\section{Hyperimmunoglobulinemia D Syndrome Variant - Seek and You Shall Find}

\section{To the Editor:}

Hyperimmunoglobulinemia D syndrome (HIDS) was first described in 1984 by Van Der Meer, et $a l^{1}$. This periodic syndrome begins in early childhood and is characterized by attacks of fever lasting 3-7 days accompanied by abdominal pain, rash, arthritis, cervical lymphadenopathy, and high urinary mevalonic acid. Between attacks, serum IgD, and to a lesser extent $\operatorname{IgA}$, is persistently elevated. The disease segregates mostly to families in the Netherlands and northern Europe. In 1999, 2 independent groups demonstrated that HIDS is caused by a recessive mutation in the mevalonate kinase $(M V K)$ gene on chromosome $12 \mathrm{q} 24^{2}$. To date, more than $50 M V K$ gene mutations have been found. Patients with HIDS can be assigned to one of 2 groups: classic type - those having mutations in the gene for MVK, or variant type - those lacking mutations ${ }^{3}$. Cases of variant HIDS may present a diagnostic challenge, frequently presenting atypical features and absence of elevated IgD. We present a case of variant HIDS in Israel, a country with low prevalence and awareness of HIDS.

A 24-year-old man of Moslem Arab origin presented to our department with 2 days of fever to $39^{\circ}-40^{\circ} \mathrm{C}$, erythematous plaque on his right malleolus, and right posterior cervical tender lymph node, $3 \times 8 \mathrm{~cm}$ in size. Laboratory results demonstrated leukocytosis $-12,000$ cells $/ \mathrm{cm}^{3}$ with

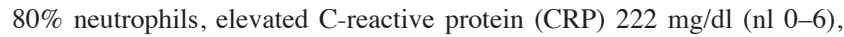
positive antinuclear antibody with nucleolar pattern, positive c-antineutrophil cytoplasmic autoantibodies and IgG anticardiolipin antibody $20 \mathrm{IU}$ (normal to 10). Urinalysis and complement concentrations were normal. Viral and bacterial serologies were negative, with cultures sterile. Computed tomography of neck, lungs, and abdomen revealed only the cervical lymphadenopathy and hepatosplenomegaly. A skin biopsy of the rash showed mild perivascular mononuclear infiltrate of the superficial dermis, with no evidence of vasculitis. The patient was treated with diclofenac, with gradual resolution of the signs - including fever, rash, and lymphadenopathy - within 7 days. Review of his past medical records at the hospital revealed 8 admissions since the age of 19 years with episodes of abdominal pain, rash, diarrhea, pleuritis, and lymphadenopathy. A previous lymph node biopsy at age 19 was consistent with a reactive pattern. A bone marrow biopsy was normal, and a previous skin biopsy showed leukocytoclastic vasculitis. In assessing him for various periodic syndromes, his immunoglobulin levels were measured and elevated $\mathrm{IgD}, 150 \mathrm{mg} / \mathrm{ml}$ (normal range $0-8 \mathrm{mg} / \mathrm{ml}$ ), and $\mathrm{IgA}, 7.54 \mathrm{mg} / \mathrm{ml}$ (normal range $0.9-3.2 \mathrm{mg} / \mathrm{ml}$ ) were noted. Gene mutation analysis of $M V K$ revealed a single V377I mutation in exon 11; MEFV and TNF receptor mutations were absent. Urine mevalonic acid was undetectable during the attack.

To the best of our knowledge this is the first case of HIDS reported from Israel and the second case in the literature of an Arab patient ${ }^{4}$. The atypical presentation of variant HIDS makes the diagnosis elusive. Simon, et al tried to characterize variant HIDS by assessing a cohort of 13 such patients in comparison to classic HIDS controls ${ }^{3}$. They found patients with variant type HIDS to have an older age at onset, longer attacks, longer remissions between attacks, less intense inflammation, and less florid clinical manifestation of serositis, arthritis, diarrhea, and lymphadenopathy. Laboratory findings, including elevated concentrations of IgA and IgD, were less evident, and $M V K$ gene mutations were found in only $76 \%$ of patients, most compound heterozygotes. A heterozygote state and negative family history in most of the patients with variant-type HIDS suggests that the occurrence of the variant type is sporadic, with a de novo mutation in only one gene in most cases. As in familial Mediterranean fever (FMF), the heterozygote state does not rule out the diagnosis, but may manifest in a less severe phenotype.

Our patient had typical features of HIDS including episodic fever, elevated IgD level, leukocytosis, elevated CRP concentration, lymphadenopathy, hepatosplenomegaly, diarrhea and rash, which suggested the diagnosis. On the other hand, atypical characteristics such as late onset, long attacks, long remissions ( $5 \mathrm{mo}$ ), cutanous vasculitis, and presence of some autoantibodies were confusing. The erythematous plaques may be confused with erysipelas-like erythema of FMF, but is actually the most common cutaneous manifestation in HIDS $^{2}$. Cutaneous leokocytoclastic vasculitis may also be confusing, yet is not uncommonly reported in HIDS. IgD immune complex-mediated pathogenesis for the skin lesions has been suggested ${ }^{5}$. There are no data in the literature on the prevalence of autoantibodies in patients with HIDS. It is suggested that the overload of $\operatorname{IgD}$ may react against self-antigens nonspecifically, thus explaining the wide autoimmune activation. This should be evaluated in a larger cohort of HIDS patients.

In conclusion, one should be aware of the possibility of the atypical presentation of variant HIDS at an adult age and consider it when evaluating patients with periodic fever, even in countries with low prevalence.

DORON RIMAR, MD; GLEB SLOBODIN, MD; ITZHAK ROSNER, MD, NINA BOULMAN, MD; MICHAEL ROZENBAUM, MD; Department of Rheumatology, Bnai Zion Medical Center, Ruth and Bruce Rappaport Faculty of Medicine, Technion, Haifa, Israel. Address correspondence to Dr. Rimar; E-mail: doronrimar@gmail.com

\section{REFERENCES}

1. van der Meer JW, Vossen JM, Radl J, van Nieuwkoop JA, Meyer CJ, Lobatto S, et al. Hyperimmunoglobulinaemia D and periodic fever: a new syndrome. Lancet 1984;1:1087-90.

2. van der Hilst JC, Bodar EJ, Barron KS, Frenkel J, Drenth JP, van der Meer JW, et al; International HIDS Study Group. Long-term follow-up, clinical features and quality of life in a series of 103 patients with hyperimmunoglobulinemia D syndrome. Medicine (Baltimore) 2008;87:301-10.

3. Simon A, Cuisset L, Vincent MF, van Der Velde-Visser SD, Delpech M, van Der Meer JW, et al. Molecular analysis of the mevalonate kinase gene in a cohort of patients with the hyper-IgD and periodic fever syndrome: its application as a diagnostic tool. Ann Intern Med 2001;135:338-43.

4. Hammoudeh M. Hyperimmunoglobulinemia D syndrome in an Arab child. Clin Rheumatol 2005;24:92-4.

5. Boom BW, Daha MR, Vermeer BJ, van der Meer JW. IgD immune complex vasculitis in a patient with hyperimmunoglobulinemia $\mathrm{D}$ and periodic fever. Arch Dermatol 1990;126:1621-4.

J Rheumatol 2011;38:10; doi:10.3899/jrheum.110518 